\title{
Biochemical Parameters in Women with Polycystic Ovary Syndrome in Ajman, UAE
}

\author{
Gomathi K', Shaafie IA', Mummigatti K², Shahid S', Sreedharan J3 \\ 'Department of Biochemistry Gulf Medical University, ${ }^{2}$ Department of Obs/Gyn, Gulf Medical College Hospital \& Research Centre, \\ ${ }^{3}$ Research Division, Gulf Medical University, Aiman, UAE
}

\begin{abstract}
Aims: Polycystic ovary syndrome (PCOS) is a common endocrine disorder affecting $5-10 \%$ of women and is a major cause of anovulatory infertility. Prevalence varies among population based on genetic and environmental factors. Etiology of PCOS remains unknown but hyperandrogenism and insulin resistance have both been associated with PCOS. The aim of this study was to measure levels of Homocysteine and other biochemical parameters in women diagnosed with PCOS attending Gulf Medical College Hospital \& Research Centre (GMCHRC), Ajman, UAE.

Methods: Young women, aged between 18 and 35 years of age, diagnosed with PCOS $(\mathrm{N}=37)$, not on any treatment, attending GMCHRC were included in the study. Biochemical parameters were measured using standard procedures. Laboratory normal reference ranges were used for comparison.

Results: $54 \%$ of the women with PCOS were overweight or obese according to the Body mass index (BMI) and $51 \%$ had a waist circumference $>88 \mathrm{~cm}$. Fasting and postprandial Glucose and Insulin levels and HOMA-IR were within the normal reference range indicating that no Insulin resistance was seen in these women. $40 \%$ of the women had a serum total Cholesterol level above $200 \mathrm{mg} / \mathrm{dL}$, while Low Density Lipoprotein (LDL) Cholesterol was above and High Density Lipoprotein (HDL) cholesterol was lower than the desirable value. Serum Triacylglycerol was within the normal reference range. Serum Testosterone, Estradiol, Prolactin Thyroid Stimulating Hormone (TSH) and Plasma Homocysteine level were found to be within the normal reference ranges. Homocysteine levels correlated with Testosterone, total Cholesterol and LDL cholesterol levels.

Conclusions: BMI was high in 54\% of the women. No Insulin resistance was seen in these patients. Hormone levels and Homocysteine were within the normal reference ranges. Dyslipidemia was observed. These findings differ from reports in literature where Insulin resistance, Hyperandrogenism and high Homocysteine levels have been associated with PCOS.
\end{abstract}

Keywords: Cholesterol, homocysteine, insulin resistance, polycystic ovary syndrome

\section{INTRODUCTION}

Polycystic ovary syndrome (PCOS) is a common endocrine disorder affecting about $5-10 \%$ of women of reproductive age worldwide. It is also estimated to be the major cause of anovulatory infertility accounting for about $73 \%$ of the cases. Variance in prevalence among populations is thought to depend on ethnic origin, race and other environmental factors on the phenotype. ${ }^{1}$

Phenotypic manifestations of the syndrome vary from patient to patient and Rotterdam diagnostic criteria for PCOS is based on the clinical identification of at least two of the three defined criteria which include (1) Oligo/anovulation (2) Clinical and/or biochemical evidence of Hyperandrogenaemia and (3) Ultrasonographic findings of polycystic ovaries. ${ }^{2,3}$
The aetiology of PCOS remains unknown but Hyperandrogenism was thought to be a main underlying factor. Now the syndrome is thought to have wider metabolic and cardiovascular implications. Insulin resistance has been found in up to $70 \%$ of women with PCOS and is a risk factor for development of type 2 Diabetes mellitus in these women. ${ }^{4}$ Altered lipid profiles and other cardiovascular disease markers like C-reactive protein and Homocysteine also suggest that PCOS could be a risk factor for development of cardiovascular disease. ${ }^{5}$

There has been very little research on the different phenotypes of PCOS or the biochemical features of PCOS in the UAE. Studies from Arabian Gulf region suggest that PCOS is the commonest cause of Hirsutism among young obese Saudi women. ${ }^{6}$ Hirsutism has been reported to be

\section{CORRESPONDENCE}

Dr. Jayadevan Sreedharan, Ph.D

Research Division

Gulf Medical University, Ajman, UAE.

Phone: +9716741333 Ext.325 Fax: +97167480779

Email: drjayadevan@gmu.ac.ae 
common among the UAE women. ${ }^{7}$ Lean women diagnosed with PCOS in the UAE have been found to respond better to metformin therapy than obese women. ${ }^{8}$

In this study, we report the biochemical and hormonal parameters in women with PCOS, attending the gynaecology clinic in the GMCHRC, Ajman, UAE.

\section{METHODS}

Study population: Women, attending the gynaecology clinic of the GMC Hospital, diagnosed with PCOS using the Rotterdam criteria ${ }^{3}$ and not on any treatment ( $N$ =37), were included in the study. Age, ethnicity was noted and anthropometric measurements like height (in $\mathrm{cm}$ ) and weight (in $\mathrm{kg}$ ) and waist circumference (in $\mathrm{cm}$ ) was measured using a standardized instrument. BMI was calculated using the formula BMI $=$ wt (in $\mathrm{kg}) / \mathrm{Ht}$ (in $\mathrm{m}){ }^{2}$

Blood samples: Venous blood samples were collected from the women after a 12 hour fast in plain tubes (for estimations in serum), tubes containing EDTA (for plasma Homocysteine) and in tubes containing Fluoride and Oxalate (for plasma Glucose estimation). Blood was also collected $2 \mathrm{~h}$ after a normal meal for postprandial glucose estimation.

Determination of Plasma Glucose (GLU), Homocysteine (Hcy), Serum Cholesterol, HDL Cholesterol (HDLc), LDL Cholesterol (LDLC) and Triacylglycerol (TG) concentrations: Plasma glucose was determined by the glucose oxidase method using a discrete chemistry analyzer (Alpha Wasserman) immediately after collection. Plasma total Homocysteine level was assayed using a Fluorescence Polarization Immuno Assay (FPIA) using the AxSYM system (Abbott Labs). Serum Cholesterol, HDLc and TG were determined by standard enzymatic kinetic procedures on the discrete chemistry analyzer (Alpha Wasserman). LDLC was calculated using the Friedwald formula.

Hormone assays: Serum Insulin, Testosterone, Estradiol, Prolactin and TSH were assayed by Chemiluminiscent immunoassay using the AxSYM system (Abbott Labs). HOMA-IR (Homeostasis model of insulin resistance) was calculated using the formula: HOMA $=$ [Fasting glucose (mmol/L) x Fasting Insulin $(\mu \mathrm{U} / \mathrm{mL})] \div 22.5$.

Data analysis: Statistical analysis was performed using PASW 18 software. Values are presented as mean \pm SD. Independent t- test was used to compare the values obtained in PCOS patients with the normal reference ranges and Pearson's correlation coefficient was used to study the relationship between the variables.

\section{RESULTS}

The women diagnosed with PCOS were between 18 and 35 years of age. Mean age was $27.08 \pm 4.02$ years. Mean $\mathrm{BMI}$ of the women was found to be $26.3 \pm 5.8 \mathrm{~kg} / \mathrm{m}^{2}$. As shown in Figure 1, 35\% (13) of the women were found to have a BMI over 25 and were overweight and 19\% (7) with $\mathrm{BMI}>30$, were obese. Mean waist circumference was $86.92 \pm 10.75 \mathrm{~cm}$ but $48.6 \%$ (18) of the women had a waist circumference above the desirable range of $<88 \mathrm{~cm}$. $35 \%$ (13) women were diagnosed with Hirsutism. Only two women $(5 \%)$ were found to be hypertensive. 10 women $(27 \%)$ had a family history of Diabetes.

As shown in Table 1, Fasting and postprandial glucose levels were $96.58 \pm 11.03$ and $107.84 \pm 26.15 \mathrm{mg} / \mathrm{dL}$ respectively. Fasting and postprandial Insulin levels were $9.72 \pm 6.01$ and $42.80 \pm 38.76 \mathrm{IU} / \mathrm{mL}$ respectively. Both Glucose and Insulin values were within the reference range. Fasting glucose to Insulin ratio and HOMA-IR, which are indices of Insulin resistance, were $12.21 \pm 5.21$ and $2.31 \pm 1.53$ respectively, were also found to be within the normal reference range.

Table 1. Plasma glucose, insulin and insulin resistance in women with $\mathrm{PCOS}(\mathrm{N}=37)$

\begin{tabular}{|c|c|c|c|}
\hline \multicolumn{2}{|c|}{ Parameter } & \multirow{2}{*}{$\begin{array}{l}\begin{array}{l}\text { Reference } \\
\text { range }\end{array} \\
70-100\end{array}$} & \multirow{2}{*}{$\begin{array}{l}\text { Values in PCOS } \\
\text { women (Mean } \\
\pm \mathrm{SD} \text { ) } \\
96.58 \pm 11.031\end{array}$} \\
\hline Plasma Glucose & Fasting & & \\
\hline (mg/dl) & Postprandial & $<140 \mathrm{mg} / \mathrm{dL}$ & $107.84 \pm 26.157$ \\
\hline \multirow{2}{*}{$\begin{array}{l}\text { Plasma Insulin } \\
\text { (IU/ml) }\end{array}$} & Fasting & $3-25$ & $9.726 \pm 6.0181$ \\
\hline & Postprandial & $10-65$ & $42.80 \pm 38.768$ \\
\hline $\begin{array}{l}\text { Glucose/Insulin } \\
\text { ratio }\end{array}$ & Fasting & $\geq 4.5$ & $12.21 \pm 5.21$ \\
\hline HOMA-IR & & $<4.0$ & $2.31 \pm 1.53$ \\
\hline
\end{tabular}

Table 2. Serum lipids and plasma homocysteine levels in women with PCOS ( $\mathrm{N}=37$ )

\begin{tabular}{lll}
\hline Parameter (units) & $\begin{array}{l}\text { Desirable/ } \\
\text { Reference } \\
\text { range }\end{array}$ & Value \\
\hline Total Cholesterol $(\mathrm{mg} / \mathrm{dL})$ & $<200$ & $192.59 \pm 31.3$ \\
LDL Cholesterol $(\mathrm{mg} / \mathrm{dL})$ & $<100$ & $123.55 \pm 27.51$ \\
HDL Cholesterol $(\mathrm{mg} / \mathrm{dL})$ & $>50$ & $42.68 \pm 8.39$ \\
Triacylglycerol $(\mathrm{mg} / \mathrm{dL})$ & $<150$ & $109.29 \pm 36.28$ \\
Triglyceride/HDL ratio & $<3.0$ & $2.69 \pm 1.09$ \\
Homocysteine $(\mu \mathrm{mol} / \mathrm{L})$ & $3.36-20.44$ & $8.49 \pm 1.98$ \\
\hline
\end{tabular}

Table 3. Serum hormone levels in women with PCOS $(\mathrm{N}=37)$

\begin{tabular}{lll}
\hline Hormone (units) & $\begin{array}{l}\text { Reference } \\
\text { range }\end{array}$ & Value \\
\hline Testosterone $(\mathrm{nmol} / \mathrm{L})$ & $<2.0$ & $1.04 \pm 0.59$ \\
Estradiol $(\mathrm{pg} / \mathrm{mL})$ & $>97.5$ & $76.35 \pm 81.43$ \\
Prolactin $(\mathrm{ng} / \mathrm{mL})$ & $64-395$ & $313.68 \pm 138.15$ \\
TSH $(\mu \mathrm{IU} / \mathrm{mL})$ & $0.49-4.67$ & $2.19 \pm 1.29$ \\
\hline
\end{tabular}

Table 4. Correlations of biochemical variables with plasma homocysteine levels

\begin{tabular}{lll}
\hline Biochemical variables & $\begin{array}{l}\text { Correlation } \\
\text { coefficient }\end{array}$ & Significance \\
\hline Cholesterol & 0.422 & $<0.05$ \\
LDL & 0.464 & $<0.05$ \\
Testosterone & 0.424 & $<0.05$ \\
\hline
\end{tabular}


Table 2 shows the lipid profile and Hcy levels in serum. Serum total Cholesterol level was found to be $192.59 \pm$ $31.3 \mathrm{mg} / \mathrm{dL}$. However $40 \%$ of the women had a serum total Cholesterol level above the desirable range $(<200 \mathrm{mg} / \mathrm{dL})$. LDLc was $123.55 \pm 27.51 \mathrm{mg} / \mathrm{dL}$ which is higher than the desirable range $(<100 \mathrm{mg} / \mathrm{dL})$. Serum HDL was $42.68 \pm$ $8.38 \mathrm{mg} / \mathrm{dL}$ which is lower than the desirable range $(>50$ $\mathrm{mg} / \mathrm{dL}$ ). Serum TG was $109.29 \pm 36.28 \mathrm{mg} / \mathrm{dL}$ which lies within the reference range.

As shown in Table 3, Serum Testosterone, Estradiol, Prolactin and TSH levels were

$1.042 \pm 0.59 \mathrm{nmol} / \mathrm{L}, 76.35 \pm 81.43 \mathrm{pg} / \mathrm{mL}, 313.68 \pm 138.15$ $\mathrm{ng} / \mathrm{mL}$ and $2.19 \pm 1.29 \mu \mathrm{lU} / \mathrm{mL}$ respectively. All values were within the normal reference ranges. However, wide variations were found in serum estradiol levels and it ranged from $0-392 \mathrm{nmol} / \mathrm{L}$.

Plasma Hcy value was $8.49 \pm 1.98 \mu \mathrm{mol} / \mathrm{L}$ which lies within the normal reference range (3.36- 20.44 umol/L) of our laboratory. Hcy levels correlated with Testosterone levels (Pearsons correlation, $r=0.424)$, Total Cholesterol $(r=0.422)$ and LDLC $(r=0.464)$. The correlation was also found to be significant, $P<0.05$ (Table 4). None of the other variables showed any relationship with each other.

\section{DISCUSSION}

$54 \%$ of the women with PCOS were found to be either overweight or obese according to their BMI and $48.6 \%$ of the women had a waist circumference above the desirable range of $<88 \mathrm{~cm}$ indicating that obesity is prevalent in these women. Our findings corroborate the high BMI and visceral adiposity that has been reported in women with PCOS ${ }^{1}$. Visceral adiposity as measured by waist circumference has been reported to be negatively associated with adiponectin levels in women with PCOS. ${ }^{9}$ The adipokines Chemerin and Leptin have been reported to be higher in women with PCOS compared to normal women. ${ }^{10-11}$

PCOS has been reported to be the commonest cause of hirsutism in the Gulf region accounting for about $90 \%$ of the cases but in our study only $35 \%$ women with PCOS were found to have hirsutism. ${ }^{6,7}$ This could be because the Rotterdam criteria used by us for diagnosis of PCOS is broader than the criteria used earlier and Hyperandrogenism is no longer a mandatory feature for diagnosis of PCOS. ${ }^{3,12}$ Multiple phenotypic presentations of the syndrome have also been reported. ${ }^{2}$

Recent studies have indicated that Insulin resistance is seen in $50-70 \%$ of women with PCOS and that it increases the risk for type 2 Diabetes. ${ }^{4,13-15}$ We did not find insulin resistance in women with PCOS. Glucose, Insulin and HOMA values were all within the normal reference ranges.
However, 10 women (27\%) had a family history of Diabetes mellitus which is similar to that reported in literature. ${ }^{16}$

Dyslipidemia has been reported in PCOS. ${ }^{5}$ Serum total cholesterol, LDLC and TG levels have been reported to be higher in PCOS compared to normal women and to be higher in obese women with PCOS compared to lean women. ${ }^{17}$ Our findings agree with the reports in literature. $40 \%$ of the women had a serum total Cholesterol and LDLC level above the desirable range and Serum HDL below the desired range. However, serum TG values were found to be within the normal reference range.

Plasma levels of total Hcy have been associated with several physiologic factors like age and gender and lifestyle factors like physical activity, smoking, folate and alcohol consumption. High blood pressure, high total cholesterol has been associated with increased Hcy levels and Hcy is now used as a risk factor for cardiovascular disease. ${ }^{18}$ Reports in literature suggest that Plasma Hcy is significantly elevated in women with PCOS.17,19 However our study shows Plasma Hcy levels in women with PCOS to be within the normal reference range. The values obtained are also comparable to those reported in literature for normal women. Plasma Hcy levels correlated with total Cholesterol, LDLC and Testosterone levels and that the correlations were significant. No significant correlation was observed between any of the other parameters studied.

Hyperandrogenism was thought to be the underlying factor for PCOS and high testosterone levels are reported in many women with PCOS. ${ }^{20}$ In our study, however, serum levels of Testosterone, Estradiol, Prolactin and TSH in women with PCOS were all within the normal reference ranges for young women. No increase in serum testosterone levels was noted even in PCOS women with hirsutism. Recent metanalysis of polycystic ovary syndrome has indicated that a number of reproductive phenotypes (subgroups) of PCOS exist based on presence or absence of ovulation, and hyperandrogenism and that they show considerable variations in adiposity and insulin resistance. ${ }^{13}$

\section{CONCLUSIONS}

$\mathrm{BMI}$ and waist circumference indicated adiposity in up to $50 \%$ of the women with PCOS. Dyslipidemia was observed in $40 \%$ of the women with PCOS. Serum total Cholesterol and LDLc level were higher and HDLc level lower than the desirable values. No Insulin resistance was observed. Serum TG, Hcy and Testosterone were all found to be within the normal reference ranges. These findings differ from reports in literature where Insulin resistance and elevated Testosterone and Hcy levels have been reported in PCOS. Since considerable phenotypic and metabolic heterogeneity in PCOS has been reported in literature, the low number of patients included in this study could be a limitation. 


\section{REFERENCES}

1. Ehrmann DA. Polycystic ovary syndrome. N Engl J Med.2005;352: 1223-36.

2. Pehlivanov B, Orbetzova M. Characteristics of different phenotypes of polycystic ovary syndrome in a Bulgarian population. Gynecol Endocrinol.2007;23(10):604-9.

3. The Rotterdam ESHRE/ASRM sponsored PCOS consensus workshop Group Revised 2003 consensus on diagnostic criteria and long term health risks related to polycystic ovary syndrome. Fertil steril.2004; 81:19-25.

4. Sattar N. Review: PCOS, insulin resistance and long-term risks for diabetes and vascular disease. Br J Diabetes Vasc Dis.2009;9:15-18.

5. Cheung LP, Ma RC, Lam PM et al. Cardiovascular risks and metabolic syndrome in Hong Kong Chinese women with polycystic ovary syndrome. Hum Reprod.2008;23(6):1431-8.

6. Al-Ruhaily AD, Malabu UH, Sulimani RA. Hirsutism in Saudi females of reproductive age : A hospital-based study. Ann Saudi Med.2008; 28:28-32.

7. Gatee OB, Al Attia HM, Salama IA. Hirsutism in the United Arab Emirates: A hospital Study. Postgrad Med J.1996;72:168-71.

8. Kumari AS, Haq A, Jayasundaram R, Abdel- Wareth LO, Al Haija SA, Alvares M. Metformin monotherapy in lean women with polycystic ovary syndrome. Reproductive Biomedicine Online.2005;10:100-4.

9. Toulis K, Goulis D, Farmakiotis D, et al. Adiponectin levels in women with polycystic ovary syndrome: A systematic review and a metaanalysis. Hum Reprod Update.2009;15:297-307.

10. Tan BK, Chen J, Farhatullah S, et al. Insulin and metformin regulate circulating and adipose tissue chemerin. Diabetes.2009;58(9): 1971-7.
11. Marciniak A, Nawrocka-Rutkowska J, Brodowska A, Sienkiewicz R, Szyd- owska I, Starczewski A. Leptin concentrations in patients with polycystic ovary syndrome before and after met-formin treatment depending on insulin resistance, body mass index and androgen con-centrations - introductory report. Folia Histochem Cytobiol.2009; $47(2): 323-8$.

12. Azziz R. Controversy In Clinical Endocrinology. Diagnosis of Polycystic Ovarian Syndrome: The Rotterdam Criteria Are Premature. J Clin Endocrinol Metab.2006;91:781-5.

13. Moran L, Teede H. Metabolic features of the reproductive phenotypes of polycystic ovary syndrome. Hum Reprod Update.2009;15(4):477-88.

14. Vrbikova J, Fanta M, Cibula D, Vondra K, Bendlova B. Impaired glucose metabolism in women with polycystic ovary syndrome. Gynecol Obstet Invest.2009;68:186-90.

15. Dabadghao P, Roberts BJ, Wang JX, et al. Glucose tolerance abnormalities in Australian women with polycystic ovary syndrome. MJA.2007;187(6):328-31.

16. Moini A, Eslami B. Familial associations between polycystic ovarian syndrome and common diseases. J Assist Reprod Genet.2009;26(2-3):123-7.

17. Yilmaz $M$, Biri A, Bukan $N$, et al. Levels of lipoprotein and homocysteine in non-obese and obese patients with polycystic ovary syndrome. Gynecol Endocrinol.2005;20(5):258-63.

18. Refsum $H$, Nurk $E$, Smith $A D$, et al. The Hordaland homocysteine study: A community-based study of homocysteine, its determinants and associations with disease. J Nutr.2006;136:1731-40.

19. Schachter M, Raziel A, Friedler S, et al. Insulin resistance in patients with polycystic ovary syndrome is associated with elevated plasma homocysteine. Hum Reprod. 2003;18 (4):721-7.

20. Homburg R. Androgen circle of polycystic ovary syndrome. Hum Reprod.2009;24(7):1548-55. 